\title{
144. CONTRIBUCIONES AL CONOCIMIENTO FLORÍSTICO DE LA COMARCA DE LAS VILLAS, JAÉN
}

\author{
Gonzalo GONZÁLEZ-JURADO
}

Recibido el 31 de agosto de 2008, aceptado para su publicación el 23 de septiembre de 2008 Publicado "on line" en enero de 2009

Contribution to the botanical knowledge of Las Villas region, Jaén

Palabras clave. Ononis viscosa L. subsp. breviflora var. pitardii Maire, Veronica serpyllifolia L. subsp. serpyllifolia, Andalucía, Jaén, Las Villas.

Key words. Ononis viscosa L. subsp. breviflora var. pitardii Maire, Veronica serpyllifolia L. subsp. serpyllifolia, Andalusia, Jaén, Las Villas.

Se aportan en esta nota nuevos datos florísticos referentes a la comarca de Las Villas, situada en la zona centro-oriental de la provincia de Jaén. Dicha comarca se extiende sobre la depresión del Guadalquivir, e incluye además la Sierra de las Villas, que forma parte del macizo montañoso de Cazorla-Segura. Es un área que, en general, ha recibido poca atención científica, especialmente en comparación con otros territorios vecinos (González-Jurado, G., 2007). Los taxones vegetales a los que se refieren dichos datos son Ononis viscosa L. subsp. breviflora (DC.) Nyman var. pitardii Maire y Veronica serpyllifolia L. subsp. serpyllifolia.

Ononis viscosa L. es una especie de amplia distribución en las regiones mediterránea y macaronésica, y que incluye un número elevado de taxones infraespecíficos (Devesa, J.A., 2000). Uno de estos taxones es O. viscosa subsp. breviflora (DC.) Nyman var. pitardii Maire, caracterizado con nitidez, dentro de este complejo grupo, por poseer dientes del cáliz estrechos que superan en longitud a la corola, por sus hojas trifoliadas y, sobre todo, por la ausencia de pelos largos eglandulares en el pedúnculo floral y en la arista que lo continúa (Devesa, J.A., 2000).
O. viscosa subsp. breviflora var. pitardii fue descrito en 1929 a partir de material norteafricano. Casi seis décadas después se detectó su presencia en Europa, concretamente en las sierras subbéticas del sur de la provincia de Córdoba (Devesa, J.A., 1986); como exclusivo de estas montañas cordobesas aparece en los compendios florísticos de Andalucía occidental (Valdés, B., Talavera, S. \& Fernández-Galiano, E., 1987) y de la Península Ibérica (Devesa, J.A., 2000). No obstante, con posterioridad a estas revisiones se localizó la planta en la provincia de Sevilla (Roales, J., 1997).

La localidad que se da a conocer en esta nota confirma que este taxón, a pesar de su aparente rareza, presenta un área de distribución extensa en el sur de España. La planta ha sido encontrada en una zona de contacto entre la depresión del Guadalquivir y el piedemonte de la Sierra de Las Villas. Los ejemplares de $O$. viscosa subsp. breviflora var. pitardii, dispersos y en escasa densidad, vegetan en taludes viarios y en márgenes de olivares, sobre margas. En la misma zona es frecuente otro taxón muy relacionado (aunque fácilmente diferenciable), O. viscosa subsp. crotalarioides (Coss.) Sirj., endémico del CS y SE de España (Devesa, J.A., 2000). 
Por su parte, Veronica serpyllifolia L. subsp. serpyllifolia presenta una distribución circumboreal muy amplia, que abarca el norte de América, Eurasia y, muy puntualmente, el norte de África (Martínez, M.M., Sánchez, J.A. \& Rico, E., inédito). En la Península Ibérica, se distribuye extensamente por la mitad septentrional (Martínez, M.M., Sánchez, J.A. y Rico, E., inédito), alcanzando el Sistema Ibérico, donde es rara y sólo aparece en las áreas más elevadas y lluviosas (Mateo, G. \& Moreno, J.M., 2004); sólo muy recientemente ha sido localizada en la provincia de Cuenca (Mateo, G. \& Moreno, J.M., 2004).

La presencia de esta planta en el macizo de Cazorla-Segura ha sido objeto de controversia. Existen citas anteriores muy antiguas (Hervier, J., 1905; Hervier J., 1906) que justifican su inclusión en catálogos florísticos locales (Benavente, A., 2005), pero no se conocían materiales de herbario que atestiguaran la presencia del taxón en la provincia de Jaén (Cano, E. \& Fernández, C., 1986). Así, la planta no es citada en este conjunto montañoso en el borrador de la revisión del género Veronica $\mathrm{L}$. para la obra Flora Iberica (Martínez, M.M., Sánchez, J.A. \& Rico, E., inédito).

La población encontrada es muy reducida, aunque no se puede precisar el número de individuos que la forman. La planta crece de forma dispersa en una pequeña zona higroturbosa que constituye la cabecera de un arroyo de montaña. La vegetación está constituida por un pastizal de mediana altura dominado por gramíneas y ciperáceas, siendo las especies más significativas Briza media L., Eleocharis palustris (L.) Roem. \& Schult., Poa pratensis L. y Plantago media L.

Las plantas que forman esta población presentan tallos radicantes en una porción corta, luego ascendentes y de 23,5-26 cm de altura, con entrenudos claramente más largos que las hojas, e inflorescencia muy alargada con cerca de 20 flores, por lo que siguiendo el esquema taxonómico propuesto en el borrador de la revisión del género para la obra Flora Iberica (Martínez, M.M., Sánchez, J.A. \& Rico, E., inédito) pertenecerían a la variedad serpyllifolia. Cabe destacar que estos ejemplares presentan abundantes pelos glandulíferos en la inflorescencia, carácter que suele atribuirse a la var. humifusa (Dicks.) Sm., aunque en la citada revisión se le resta a dicha pilosidad importancia taxonómica (Martínez, M.M., Sánchez, J.A. \& Rico, E., inédito).

\section{Material estudiado}

Ononis viscosa L. subsp. breviflora (DC.) Nyman var. pitardii Maire

ESPAÑA. Jaén: Villanueva del Arzobispo, pr. antigua cooperativa San Miguel, 30S 501050 4226470, $700 \mathrm{~m}$, taludes y márgenes del olivar, sobre margas, 12-V-2008, G. González-Jurado, MGC. 68804.

Veronica serpyllifolia L. subsp. serpyllifolia ESPAÑA. Jaén: Iznatoraf, Cañada Somera, 16-VI-2008, 30S 5137004217800 , $1650 \mathrm{~m}$, pastizales sobre suelos higroturbosos. G. González-Jurado, MGC. 68805.

\section{BIBLIOGRAFÍA}

BENAVENTE NAVARRO, A. -2005- Revisión del Catálogo de Fanerógamas del Parque Natural de las Sierras de Cazorla, Segura y Las Villas. Taller de Ecología - Ecologistas en Acción, Linares.

CANO CARMONA, E. y C. FERNÁNDEZ LÓPEZ -1986- Distribución de las escrofulariáceas en la provincia de Jaén. Blancoana 4: 83-110.

DEVESA, J.A. -1986- Notas taxonómicas y corológicas sobre la flora de Andalucía Occidental: Nota 192. Ononis viscosa subsp. breviflora var. pitardii. Lagascalia 14(1): 151152.

DEVESA, J.A. -2000- Ononis L. in S. Castroviejo $\&$ al., eds., Flora Iberica vol. VII(II). Real Jardín Botánico, Madrid.

GONZÁLEZ-JURADO, G. -2007- Aportación al 
conocimiento de los plecópteros euholognatos (Plecoptera: Euholognatha) de la Sierra de Las Villas (Jaén, España). Boletín de la Sociedad Entomológica Aragonesa 41:453-454.

HERVIER, J. -1905-Bull. Acad. Int. Géogr. Bot. Le Mans 15: 1-32.

HERVIER, J. -1906-Bull. Acad. Int. Géogr. Bot. Le Mans 16: 201-232.

MARTÍNEZ ORTEGA, M.M., J.A SÁNCHEZ AGUDO y E. RICO (inédito) Veronica L. in S. Castroviejo et al., eds., Flora Iberica vol. XIII. Real Jardín Botánico, Madrid. [Borrador de la síntesis del género, disponible en www. rjb.csic.es]

MATEO SANZ, G. y J.M. MORENO VALDEOLIAS -2004- "Nuevos datos sobre la flora de la provincia de Cuenca, XX" Flora Montiberica 26: 3-6.

ROALES, J. -1997- Contribución al conocimiento de la flora de Sevilla I: novedades corológicas para la provincia. Lagascalia 20(1): 129-150.

TRIANO, E. -1998- Flora del Subbético cordobés. Catálogo, recursos y curiosidades. Ayto. de Rute, Diputación Prov. de Córdoba, Córdoba.

VALDÉS, B., S. TALAVERA y E. FERNÁNDEZGALIANO -1987- Flora Vascular de Andalucía Occidental. Ed. Ketres, Sevilla.

Dirección del autor. C/ Cristóbal Millán 22, C.P. 23300 Villacarrillo (Jaén, España).

ggonjur@mixmail.com

\section{LITTORELLA UNIFLORA EN LAS LAGUNAS DE MOGUER (PARQUE NATURAL DE DOÑANA, SW PENINSULA IBÉRICA)}

\section{Pablo GARCÍA MURILLO* y Manuela PALACIOS GONZÁLEZ}

Recibido el 27 de marzo de 2009, aceptado para su publicación el 29 de junio de 2009

Publicado "on line" en octubre de 2009

\section{Littorella uniflora in Moguer Pond Complex (Doñana Natural Park, SW Iberian Peninsula)}

Palabras clave. Littorella, plantas acuáticas, flora acuática, Andalucía, Doñana, Peninsula Ibérica.

Key words. Littorella, aquatic plants, aquatic flora, Donana, Andalusia, Iberian Peninsula.

Huelva. Moguer. Lagunas de Moguer. Pastizales higroturbosos en el borde de una laguna. 29SPB9914, 22/3/2009. P. García Murillo \& M. Palacios (SEV237734).

En el año 1993 aparecía una nota en la revista Lagascalia firmada por Hellmann \&
Hellmann donde se informaba sobre el hallazgo de Littorella uniflora (L.) Ascherson en una zona del manto eólico litoral onubense, en el SW de Andalucía. Dicho hallazgo resultaba de gran interés desde el punto de vista corológico, ya que ampliaba considerablemente el área 